ORIGINAL ARTICLE

\title{
Distribution of HPV infection and tumour markers in cervical intraepithelial neoplasia from cone biopsies of Mozambican women
}

\author{
C Carrilho, L Cirnes, M Alberto, L Buane, N Mendes, L David
}

J Clin Pathol 2005;58:61-68. doi: 10.1136/icp.2004.020552

See end of article for authors' affiliations .....................

Correspondence to: Dr L David, Instituto de Patologia e Imunologia Molecular da Universidade do Porto (IPATIMUP), Rua Roberto Frias, s/n, 4200, Porto, Portugal;

leonor.david@ipatimup.pt

Accepted for publication 10 August 2004
Aims: To evaluate human papillomavirus (HPV) infection in whole cervical cone specimens with cervical intraepithelial neoplasia (CIN). In addition, to evaluate the relation between the presence of CIN lesions and HPV infection and the expression of Ki-67, p53, cytokeratins, Gp230 glycoprotein, and simple mucintype carbohydrates.

Methods: Cervical cone specimens from five patients with CIN were studied. For each specimen, serial sections encompassing the whole cone were collected (52 samples). HPV infection and HPV types were detected by the polymerase chain reaction and enzyme immunoassay. The expression of Ki-67, p53, cytokeratins, Gp230, and simple mucin-type carbohydrates was examined immunohistochemically.

Results: All cases showed high risk HPV types, namely types 16, 33, 35, and 58. Four of the five patients were infected by multiple viral types. HPV-58 was always seen in CIN III, whereas HPV-35 was more frequent in CIN I. The expression of $\mathrm{Ki}-67$ and p53 was higher in CIN III lesions. The expression of cytokeratins 8 and 17 showed complete or almost complete overlap with CIN III. Altered expression of Gp230, Tn, and sialyl-T was often seen in all grades of CIN.

Conclusions: When whole cervical cone specimens are evaluated the rate of multiple HPV infection is very high. The expression of cytokeratins 8 and 17 is a useful marker of CIN III.

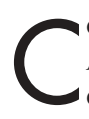
ervical carcinoma is a major cause of cancer death in Africa. The strong causal association between cervical carcinoma and infection by human papillomavirus (HPV) is well established, and it was recently proposed that HPV infection is a necessary cause of cervical cancer development. ${ }^{1}$ Both our group, ${ }^{2}$ and that of Castellsague et al, ${ }^{3}$ have previously described the profile of HPV infection in Mozambican women. ${ }^{23}$ In both studies, the types of HPV identified coincided with those previously described in other sub-Saharan countries, with a high prevalence of HPV types $16,31,33,35$, and $58 .^{2-6}$

With the improvement of methods to detect HPV at the molecular level there is increasing evidence of the presence of HPV in cervical intraepithelial neoplasia (CIN) lesions. ${ }^{78}$ The detection of multiple infections has also increased, probably because of the higher sensitivity of the techniques that are currently used. ${ }^{9-11}$

\section{"Cervical carcinoma is a major cause of cancer death in Africa"}

Very few studies have explored the relation between the topographical distribution of HPV infection and HPV types and the different grades of CIN lesions in the uterine cervix. $^{12}{ }^{13}$ To the best of our knowledge, no studies relating the presence or absence of HPV infection and HPV types in CIN lesions, which have thoroughly evaluated the whole cervix, have been undertaken.

CIN and HPV infection are accompanied by several alterations in molecular structures that are expressed de novo or are lost. The putative usefulness of these markers for diagnostic and prognostic purposes is still controversial, and the results so far obtained are frequently conflicting.

In our present study, using serial samples from total inclusions of whole cervixes with intraepithelial lesions, we aimed to determine the distribution and types of HPV infection, in addition to the association between infection and the presence of different grades of intraepithelial lesion and with different markers associated with infection/lesions in previous studies (Ki-67, p53, cytokeratin 8 (Ck8), Ckl0, Ck13, Ck17, Gp230 glycoprotein, simple mucin-type carbohydrates Tn, sialyl-Tn, T, and sialyl-T).

\section{METHODS}

\section{Tissue}

Cervical cone specimens from five consecutive patients with CIN diagnosed at the department of pathology of the Maputo Central Hospital in Mozambique were studied. For each cone specimen, several samples obtained by serial radial sections encompassing the whole cone were collected, varying from seven to 13 samples for each cone-52 samples in total. All CIN lesions were located in the transformation zone. Figures 1-5 depict the location of the lesions, and serial sections are numbered clockwise starting from the anterior lip of the cervix at 12 hours (section 1 in all cases). All samples were fixed in $10 \%$ formalin, embedded in paraffin wax, and cut into $4 \mu \mathrm{m}$ thick sections. Haematoxylin and eosin stained sections were used to grade CIN lesions according to the World Health Organisation recommendations. ${ }^{14}$

\section{Immunohistochemistry}

Immunohistochemistry was performed to study the expression of Ck8, Ck10, Ck13, Ck17, Gp230 glycoprotein, p53, Ki67, and mucin-type carbohydrates-Tn, sialyl-Tn, T, and sialyl-T. The detection of the sialyl-T antigen was performed using antibodies directed at the $\mathrm{T}$ antigen, after treating the sections with neuraminidase.

Abbreviations: $\mathrm{CIN}$, cervical intraepithelial neoplasia; $\mathrm{Ck}$, cytokeratin; EIA, enzyme immunoassay; HPV, human papillomavirus; PCR, polymerase chain reaction 
Table 1 Monoclonal antibodies, isotypes, working dilutions, and sources

\begin{tabular}{|c|c|c|c|c|}
\hline Antigen & Antibody & Isotype & Dilution & Source \\
\hline Ck8 & NCL-Ck8-TS1 & $\lg G 1$ & $1 / 100$ & Novocastra Laboratories, Newcastle, UK \\
\hline Ck10 & $\mathrm{NCL}-\mathrm{Ck} 10$ & lgG1, $\kappa$ light chain & $1 / 50$ & Novocastra Laboratories \\
\hline Ck13 & $\mathrm{NCL}-\mathrm{Ck} 13$ & $\lg G 1$ & $1 / 200$ & Novocastra Laboratories \\
\hline $\mathrm{Ck} 17$ & $\mathrm{NCL}-\mathrm{Ck} 17$ & $\lg G 2 b$ & $1 / 20$ & Novocastra Laboratories \\
\hline Tn & HB-Tn & $\lg M$ & $1 / 15$ & H Clausen, S Hakamori, unpublished \\
\hline Sialyl-Tn & HB-STn & $\lg G 1$ & $1 / 8$ & Kjeldsen and colleagues ${ }^{15}$ \\
\hline $\mathrm{T}$ & HB-T & $\lg M$ & $1 / 10$ & Clausen and colleagues $^{16}$ \\
\hline Gp230 & PANH4 & $\lg M$ & $1 / 10$ & Nielsen and colleagues ${ }^{17}$ \\
\hline p53 & DO7 & $\lg G 2 b$ & $1 / 50$ & Novocastra Laboratories \\
\hline $\mathrm{Ki}-67$ & MM1 & $\lg G 1$ & $1 / 100$ & Novocastra Laboratories \\
\hline
\end{tabular}

Ck, cytokeratin.

\section{Antibodies}

Table 1 lists the mouse monoclonal antibodies used, their respective isotypes, working dilutions, and sources.

\section{Immunostaining}

Serial sections ( $4 \mu \mathrm{m}$ thick) from $10 \%$ formalin fixed, paraffin wax embedded material were used for immunostaining. Immunohistochemistry was carried out according to the avidin-biotin-peroxidase complex method ${ }^{18}$ after dewaxing. Before immunostaining for Ckl0, the specimens were trypsin digested at room temperature in Tris buffered saline. For the remaining keratins-Ck8, $\mathrm{Ckl3}$, and $\mathrm{Ck17}$-and for p53 and Ki-67, specimens were submitted to high temperature (pressure cooker) using a $10 \mathrm{mM}$ sodium citrate buffer solution, $\mathrm{pH}$ 6. Sections to be treated with neuraminidase were preincubated with type VI neuraminidase from Clostridium perfringens (Sigma, Poole, Dorset, UK), diluted in $0.2 \mathrm{M}$ sodium acetate buffer, $\mathrm{pH} 5.5$, to a final concentration

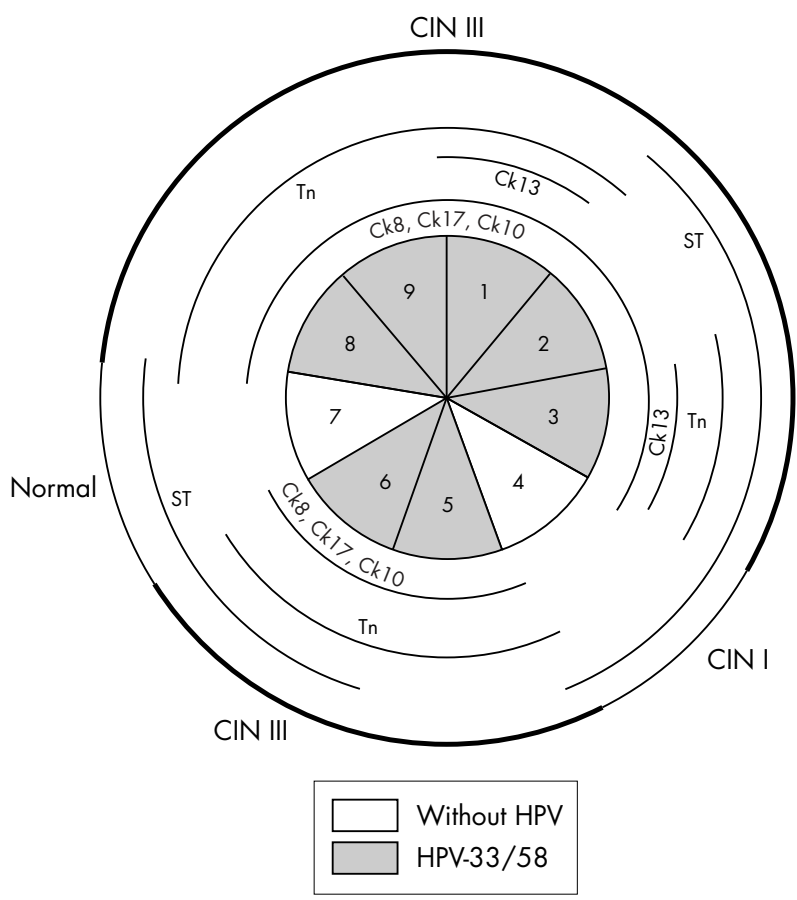

Figure 1 Schematic representation of case 1, including the absence or presence of human papillomavirus (HPV) and HPV types in the nine sections. Lesions in the whole specimen are outlined in the outer circle, with cervical intraepithelial neoplasia (CIN) III lesions marked with a thicker line. Different cytokeratins (Cks) and glycoprotein markers are outlined in the inner circles whenever their pattern of expression was abnormal. of $0.1 \mathrm{U} / \mathrm{ml}$. In all sections, endogenous peroxidase was blocked by incubation in $0.3 \% \mathrm{H}_{2} \mathrm{O}_{2}$ in methanol for 10 minutes. Sections were incubated for 20 minutes with normal non-immune serum to eliminate non-specific staining. Excess normal serum was removed from the slides. The sections were then incubated for 30 minutes with the primary antibodies (dilutions specified in table 1), at room temperature for all Cks, p53, and Ki-67, and overnight for simple mucin-type carbohydrate antigens and Gp230 glycoprotein. This step was followed by incubation with a $1 / 200$ dilution of biotin labelled antimouse secondary antibody (Dako, Copenhagen, Denmark) for 30 minutes and avidinbiotin-peroxidase complex for a further 30 minutes.

Careful rinses with Tris buffered saline were performed between each step of the procedure. The slides were then treated with 3'3-diaminobenzidinetetrahydrochloride, counterstained with Mayer's haematoxylin, dehydrated, and mounted.

All series included positive controls. Negative controls were carried by omission of the primary antibodies. The presence

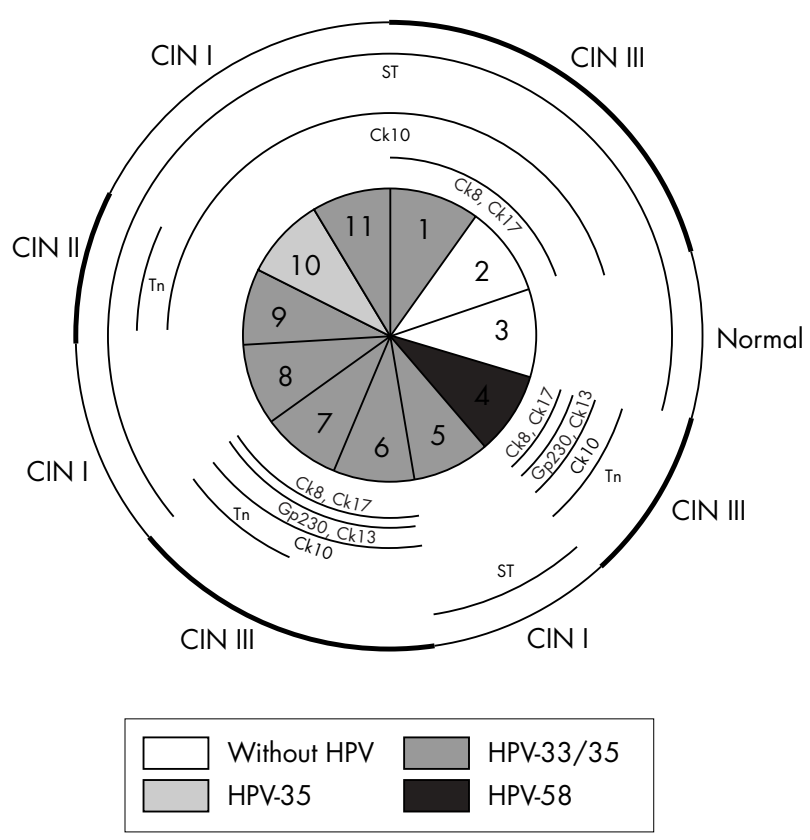

Figure 2 Schematic representation of case 2, including the absence or presence of human papillomavirus (HPV) and HPV types in the 11 sections. Lesions in the whole specimen are outlined in the outer circle, with cervical intraepithelial neoplasia (CIN) II and III lesions marked with a thicker line. Different cytokeratins (Cks) and glycoprotein markers are outlined in the inner circles whenever their pattern of expression was abnormal. 


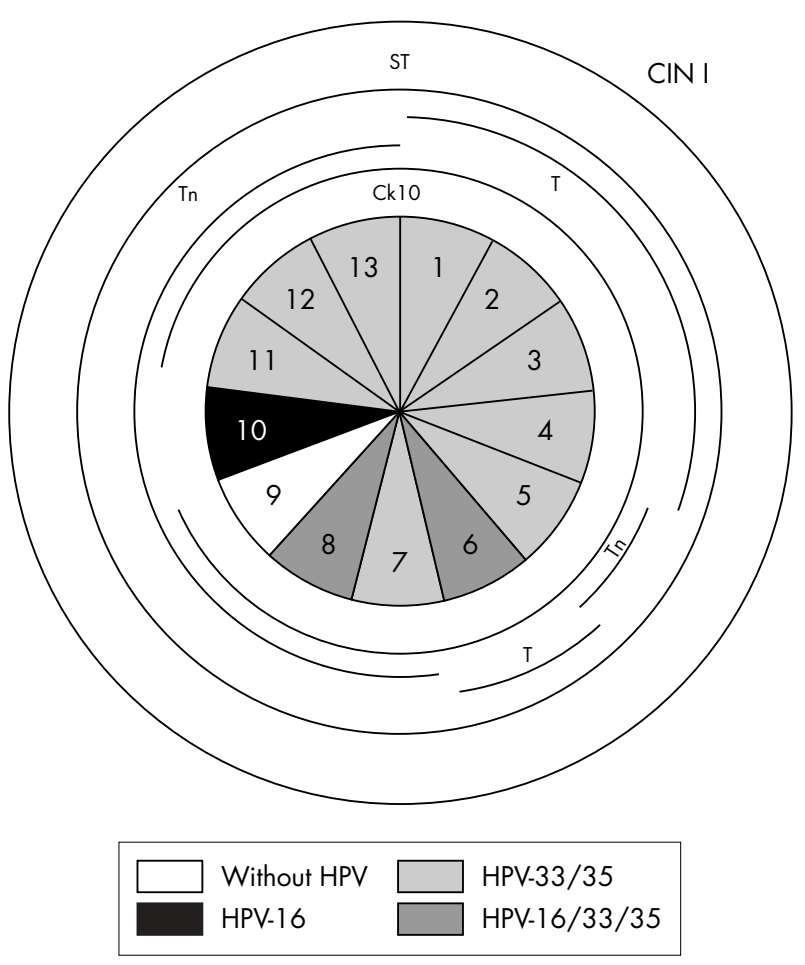

Figure 3 Schematic representation of case 3, including the absence or presence of human papillomavirus (HPV) and HPV types in the 13 sections. Lesions of cervical intraepithelial neoplasia (CIN) I were observed in the whole specimen and are outlined in the outer circle. Different cytokeratins (Cks) and glycoprotein markers are outlined in the inner circles whenever their pattern of expression was abnormal.

of sialyl-T antigen in red blood cells was used as an internal positive control for the sections subjected to neuraminidase.

\section{Abnormal immunostaining profiles and scoring of the staining}

To detect abnormal expression, the expression of Cks, mucintype carbohydrates, and Gp230 glycoprotein was compared with the normal profile of expression defined in previous studies (table 2). For p53 and Ki-67 the number of positive cells was obtained by counting 100 cells in each section.

\section{Molecular study for HPV detection}

Sections for the detection and characterisation of HPV were obtained between two haematoxylin and eosin stained sections to control for the presence of representative sampling of the lesions. To avoid contamination, the section knife was replaced after cutting each section, and an empty paraffin wax block was used alternately. All negative samples were re-tested after DNA extraction and polymerase chain reaction (PCR) by cutting new sections, followed by a haematoxylin and eosin stained section to control for the presence of the lesion.

\section{DNA preparation}

DNA was extracted and purified according to standard techniques and the protocol used in our previous study. ${ }^{2}$ Each tissue section was digested with $10 \mathrm{mg} / \mathrm{ml}$ proteinase $\mathrm{K}$ in a buffered solution. For the PCR reaction $10 \mu \mathrm{l}$ aliquots of DNA were used.

\section{PCR}

To check the quality of the target DNA all samples were tested by PCR using $\alpha$ actin specific primers to amplify a

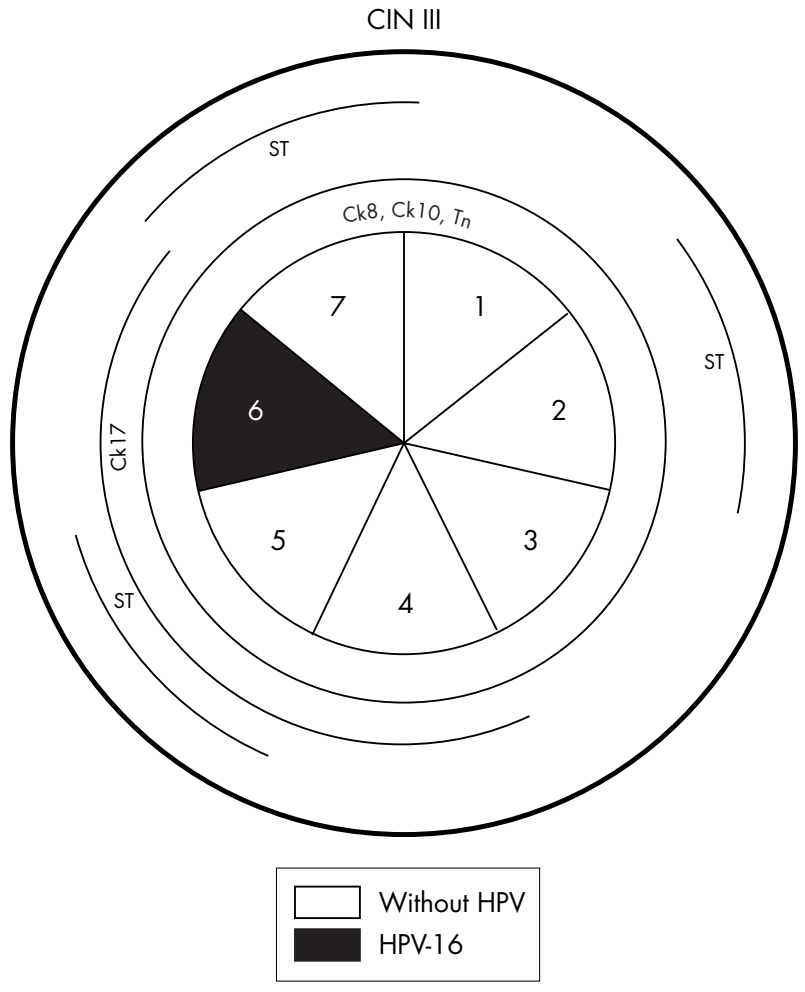

Figure 4 Schematic representation of case 4, including the absence or presence of human papillomavirus (HPV) and HPV types in the seven sections. Lesions of cervical intraepithelial neoplasia (CIN) III were observed in the whole specimen and are outlined in the outer circle. Different cytokeratins (Cks) and glycoprotein markers are outlined in the inner circles whenever their pattern of expression was abnormal.

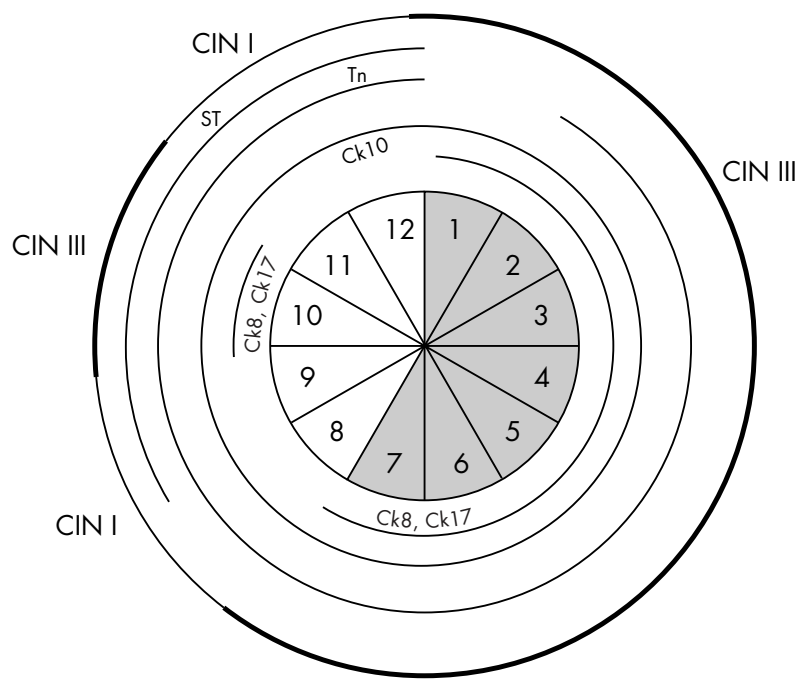

Without HPV

HPV-33/58

Figure 5 Schematic representation of case 5, including the absence or presence of human papillomavirus (HPV) and HPV types in the 12 sections. Lesions in the whole specimen are outlined in the outer circle, with cervical intraepithelial neoplasia (CIN) III lesions marked with a thicker line. Different cytokeratins (Cks) and glycoprotein markers are outlined in the inner circles whenever their pattern of expression was abnormal. 
200 bp region of the $\alpha$ actin gene (forward primer, ctt cct ggg cat gga gtc; reverse primer, cgc tca gga gga gca atg at). Specimens that showed successful amplification of $\alpha$ actin sequences were subjected to HPV DNA detection, with the general primers GP5+/bio-GP6+ (Eurogentec, Liege, Belgium), as described previously, ${ }^{21}$ to amplify a $150 \mathrm{bp}$ fragment of the Ll region of HPV. The GP5+/bio-GP6+ primers were used at a concentration of $25 \mathrm{pmol}$ each. The mixture was incubated for four minutes at $94^{\circ} \mathrm{C}$ for DNA denaturation. Forty cycles of amplification were carried out using a PCR processor (Perkin-Elmer 2400; Perkin Elmer, Foster City, California, USA). Each cycle included denaturation at $94^{\circ} \mathrm{C}$ for one minute, followed by primer annealing at $40^{\circ} \mathrm{C}$ for two minutes, and chain elongation at $72^{\circ} \mathrm{C}$ for 1.5 minutes. To ensure complete extension of the amplified DNA the final elongation step was prolonged by 10 minutes. Aliquots $(10 \mu \mathrm{l})$ of each PCR product were run on $2 \%$ agarose gel electrophoresis gels and stained with ethidium bromide $(2 \mathrm{mg} / \mathrm{ml})$. Aliquots $(5 \mu \mathrm{l})$ of the biotinylated GP-PCR products were used for enzyme immunoassay (EIA) for PCR based HPV detection and typing.

\section{Enzyme immunoassay}

EIA was performed using oligoprobes type specific for HPV types 16, 18, and 35, a cocktail specific for six low risk HPVs $(6,11,40,42,43$, and 44), and another cocktail specific for 11 high risk HPVs (31, 33, 39, 45, 51, 52, 56, 58, 59, 66, and 68). In the samples positive for the high risk HPV cocktail, a second EIA was performed using oligoprobes type specific for HPV types 31, 33, 45, 52, and 58.

EIA was carried our according to the literature ${ }^{21} 22$ and following the protocol used in our previous study. ${ }^{2}$ Briefly, EIA was performed in streptavidin coated microtitre plates (Boehringer-Mannheim, Mannheim, Germany). Washings were performed at room temperature for each step. The EIA procedure used biotinylated PCR products captured in streptavidin coated microtitre wells. Subsequently, wells were washed and the captured DNA was denatured by alkaline treatment at room temperature. Wells were washed again, followed by hybridisation with digoxigenin labelled oligoprobes (Eurogentec) specific for the HPV types described above $(10 \mathrm{pmol} / \mathrm{ml}$ each in $50 \mu \mathrm{l}$ of hybridisation buffer solution). Unbound probe was removed by washing. The hybrids were then detected using $50 \mu \mathrm{l}$ of antidigoxigenin antibodies conjugated with alkaline phosphatase (Boehringer-Mannhein; $150 \mathrm{U} / 200 \mu \mathrm{l}$ ), after removing the unbound polyclonal antibodies; p-nitrophenyl phosphate (Sigma) was added as substrate.

After incubation at $37^{\circ} \mathrm{C}$ for one hour and overnight, the optical density was read with a Hyperion Micro Reader 3, as described previously. ${ }^{21}$

Table 2 Normal profile of expression of $\mathrm{Ck}$ and mucin markers in human cervical epithelium defined previously ${ }^{17} 1920$

\begin{tabular}{ll}
\hline Markers & Expression \\
\hline Ck8 & Absent \\
Ck17 & Absent \\
Ck10 & Present (superficial and intermediate layers) \\
Ck13 & Present (suprabasal layers) \\
Gp230 & Present (suprabasal layers) \\
Tn & Present (superficial and intermediate layers) \\
Sialyl-Tn & Present (suprabasal layers) \\
T & Absent \\
Sialyl-T & Absent \\
\hline Ck, cytokeratin. & \\
\hline
\end{tabular}

\section{RESULTS}

HPV infection, CIN lesions, and expression of p53 and $\mathrm{Ki}-67$

We studied a total of 52 samples from the five patients, encompassing the whole cervical cone specimen from each patient (seven to 13 samples for each cone specimen) (figs $1-5$ ).

All the patients showed HPV infection with high risk HPV types. Low risk HPV types were never detected. Four of the five patients were infected with multiple HPV types. We found coinfection by HPV-33 and HPV-58 in two patients; by HPV-33, HPV-35, and HPV-58 in one patient; by HPV-16, HPV-33, and HPV-35 in one patient; and by HPV-16 alone in another patient (table 3 ). HPV-33 was seen in four cases, HPV-58 was seen in three cases, and HPV-16 and HPV-35 were seen in two cases each (table 3 ).

Taking all samples together, we found coinfection with multiple HPV types in 32 of 52 samples ( 32 of $36 \mathrm{HPV}$ positive samples). Seventeen of all 27 samples ( 17 of $19 \mathrm{HPV}$ positive samples) with CIN III lesions showed infection with multiple HPV types (tables 3 and 4 ). Table 4 shows the association between the presence or absence of CIN lesions of variable grade according to the presence or absence of HPV and of HPV types/combinations in all the samples from the five patients. All the HPV positive samples had CIN. However, 14 of the $16 \mathrm{HPV}$ negative samples also had CIN lesions. HPV-58 alone or in combination with HPV-33 was always found in CIN III lesions. HPV-35 alone or in combination with HPV-33 was more frequently seen in CIN I lesions (table 4).

The percentage of cells positive for Ki-67 or p53 was similar in HPV negative and positive samples and in samples with different HPV types (table 5). A higher proportion of Ki-67 and p53 positive cells was seen in CIN III lesions (table 5) (fig 6A).

\section{Patterns of expression of Cks, Gp230 glycoprotein, and simple mucin-type carbohydrates in the whole specimen cone sets}

Abnormal expression of Sialyl-Tn was not detected.

- Case 1 (fig 1): abnormal expression of Ck8 (fig 6B), Ck17, Ck10, and Tn antigen largely coincides with both CIN III lesions and HPV infection. Ck13 (fig 6C, D) and Gp230 glycoprotein (fig 6E, F) are rarely expressed with an abnormal pattern. Abnormal expression of Sialyl-T antigen does not co-localise with either CIN lesions or the presence of HPV.

- Case 2 (fig 2): abnormal expression of Ck8, Ck10, and Ck17 largely coincides with both CIN III lesions and HPV infection. An exception is seen in section 2, with abnormal expression of the three markers in the absence of HPV. Ck10, in contrast to $\mathrm{Ck} 8$ and $\mathrm{Ck} 17$, is also expressed in CIN I (sections 10 and 11) and CIN II lesions (section 9). Ck13,Gp230 glycoprotein, and Tn antigen are rarely expressed with an abnormal pattern. Abnormal expression of Sialyl-T antigen does not co-localise with either CIN lesions or the presence of HPV.

- Case 3 (fig 3): abnormal expression of Ck10, Tn, T, and Sialyl-T is seen in almost all the tissue sections.

- Case 4 (fig 4): abnormal expression of Ck8, Ck10, and Tn antigen is seen in all sections with CIN III lesions, independent of the presence of HPV. Both Ckl7 and sialyl-T antigen have a more limited abnormal expression pattern.

- Case 5 (fig 5): abnormal expression of Ck8 and Ckl7 coincides with both CIN III lesions and HPV infection. Cklo and Tn antigen are abnormally expressed in almost 
Table 3 Presence or absence of CIN according to the presence or absence of HPV and HPV types/combinations in all samples of whole cones from each patient

\begin{tabular}{|c|c|c|c|}
\hline Case (no of samples) & No of samples/sections* & Histology & HPV type \\
\hline \multirow[t]{3}{*}{ Case $1(n=9)$} & $1 /$ section 7 & Normal & Negative \\
\hline & $1 /$ section 4 & CIN I & Negative \\
\hline & $7 /$ sections $1,2,3,5,6,8,9$ & CIN III & 33,58 \\
\hline \multirow[t]{7}{*}{ Case $2(n=11)$} & $1 /$ section 3 & Normal & Negative \\
\hline & $3 /$ sections $5,8,11$ & $\mathrm{CIN} \mathrm{I}$ & 33,35 \\
\hline & $1 /$ section 10 & $\mathrm{CIN} \mathrm{I}$ & 35 \\
\hline & $1 /$ section 9 & $\mathrm{CIN} \|$ & 33,35 \\
\hline & $3 /$ sections $1,6,7$ & CIN III & 33,35 \\
\hline & $1 /$ section 4 & CIN III & 58 \\
\hline & 1/section 2 & $\mathrm{CIN}$ III & Negative \\
\hline \multirow[t]{4}{*}{ Case $3(n=13)$} & $9 /$ sections $1,2,3,4,5,7,11,12,13$ & $\mathrm{CINI}$ & 33,35 \\
\hline & $2 /$ sections 6,8 & $\mathrm{CINI}$ & $16,33,35$ \\
\hline & $1 /$ section 10 & $\mathrm{CINI}$ & 16 \\
\hline & $1 /$ section 9 & $\mathrm{CINI}$ & Negative \\
\hline \multirow[t]{2}{*}{ Case $4(n=7)$} & $6 /$ sections $1,2,3,4,5,7$ & CIN III & Negative \\
\hline & $1 /$ section 6 & CIN III & 16 \\
\hline \multirow[t]{3}{*}{ Case $5(n=12)$} & $4 /$ sections $8,9,11,12$ & $\mathrm{CIN} \mathrm{I}$ & Negative \\
\hline & $7 /$ sections $1,2,3,4,5,6,7$ & $\mathrm{CIN}$ III & 33,58 \\
\hline & $1 /$ section 10 & CIN III & Negative \\
\hline
\end{tabular}

*Sections are numbered in figs $1-5$.

$\mathrm{CIN}$, cervical intraepithelial neoplasia; HPV, human papillomavirus.

Table 4 Presence or absence of CIN according to the presence or absence of HPV and HPV types/combinations in all the sections from the five patients

\begin{tabular}{|c|c|c|c|c|}
\hline & Normal cervix (n/\%) & CIN I (n/\%) & CIN II (n/\%) & CIN III (n/\%) \\
\hline HPV negative $(n=16)$ & $2 / 12.5$ & $6 / 37.5$ & $0 / 0$ & $8 / 50.0$ \\
\hline HPV-33/58 $(n=14)$ & $0 / 0$ & $0 / 0$ & $0 / 0$ & $14 / 100$ \\
\hline HPV-33/35 $(n=16)$ & $0 / 0$ & $12 / 75.0$ & $1 / 6.3$ & $3 / 18.7$ \\
\hline HPV-16/33/35 (n=2) & $0 / 0$ & $2 / 100$ & $0 / 0$ & $0 / 0$ \\
\hline HPV-16 $(n=2)$ & $0 / 0$ & $1 / 50.0$ & $0 / 0$ & $1 / 50.0$ \\
\hline HPV-35 $(n=1)$ & $0 / 0$ & $1 / 100$ & $0 / 0$ & $0 / 0$ \\
\hline HPV-58 $(n=1)$ & $0 / 0$ & $0 / 0$ & $0 / 0$ & $1 / 100$ \\
\hline Total HPV positive $(n=36)$ & $0 / 0$ & $16 / 44.4$ & $1 / 2.8$ & $19 / 52.8$ \\
\hline
\end{tabular}

all the tissue sections. Sialyl-T antigen is rarely expressed with an abnormal pattern.

\section{Relation between HPV infection and CIN lesions in the whole specimen cone sets}

In most cases, CIN lesions coincided with the presence of HPV. In cases 1, 2, 4, and 5 we saw CIN lesions in the absence of detectable HPV. We never detected HPV in sections without CIN lesions (cases 1 and 2).

\section{DISCUSSION}

Our present study showed a high frequency of HPV infection by multiple viral types in cervical specimens with CIN lesions. In fact, infection by a single viral type (HPV-16) was found in only one case. All the viral types identified were high risk HPVs and included HPV types 16, 33, 35, and 58.

To the best of our knowledge, our study is the first to evaluate thoroughly the presence and viral types of HPV in whole cone specimens. This is probably the reason why we found such a high frequency of multiple infections (four of five cases). The prevalence of HPV infection by multiple viral types reported in the literature varies from $24.5 \%{ }^{11}$ to $40-$ $50 \% .^{3}{ }^{9} 1023$

With the improved methods to detect HPV at the molecular level there is increasing evidence of the presence of HPV in CIN lesions. ${ }^{78}$ The detection of multiple infections has also increased, probably because of the higher sensitivity of the techniques that are currently used..$^{9-11}$ We used a GP5+/bioGP6+ PCR-EIA method, which has a high sensitivity and specificity for HPV/HPV type detection, as demonstrated in previous studies. $^{21} 22$

Two of the five patients were human immunodeficiency virus negative when the cone specimen was taken and both had multiple infections (cases 3 and 5), so that we can assume that the high rate of multiple infections in our cases is not the result (at least exclusively) of human immunodeficiency virus infection.

Table 5 Percentage of $\mathrm{Ki}-67$ and p53 positive cells according to the presence or absence of HPV and the different HPV types/combinations and CIN lesions in all sections from the five cases

\begin{tabular}{lll}
\hline & Ki-67 & p53 \\
\hline HPV negative $(n=16)$ & $23.9(23.6)$ & $6.5(12.4)$ \\
HPV positive $(n=36)$ * & $25.9(13.7)$ & $3.1(5.0)$ \\
HPV-33/58 $(n=14)$ & $26.1(10.7)$ & $4.9(5.2)$ \\
HPV-33/35 $(n=14)$ & $23.0(16.2)$ & $0.8(1.5)$ \\
HPV-16/33/35 $(n=2)$ & $29.0(1.4)$ & 0 \\
HPV-16 $(n=2)$ & $35.5(27.6)$ & $10.0(14.1)$ \\
HPV-58 $(n=1)$ & 24 & 3 \\
Normal $(n=2)$ & $0.5(0.7)$ & $0.5(0.7)$ \\
CIN I $(n=20)$ & $19.3(13.7)$ & $1.5(2.7)$ \\
CIN III $(n=27)$ & $31.6(17.4)$ & $6.5(10.4)$ \\
\hline
\end{tabular}

Values are mean (SD).

*Three HPV positive sections (1 with HPV- 35 and 2 with HPV-33 and HPV-35) could not be used for Ki-67 and p53 evaluation because of a lack of material.

$\mathrm{CIN}$, cervical intraepithelial neoplasia; HPV, human papillomavirus. 

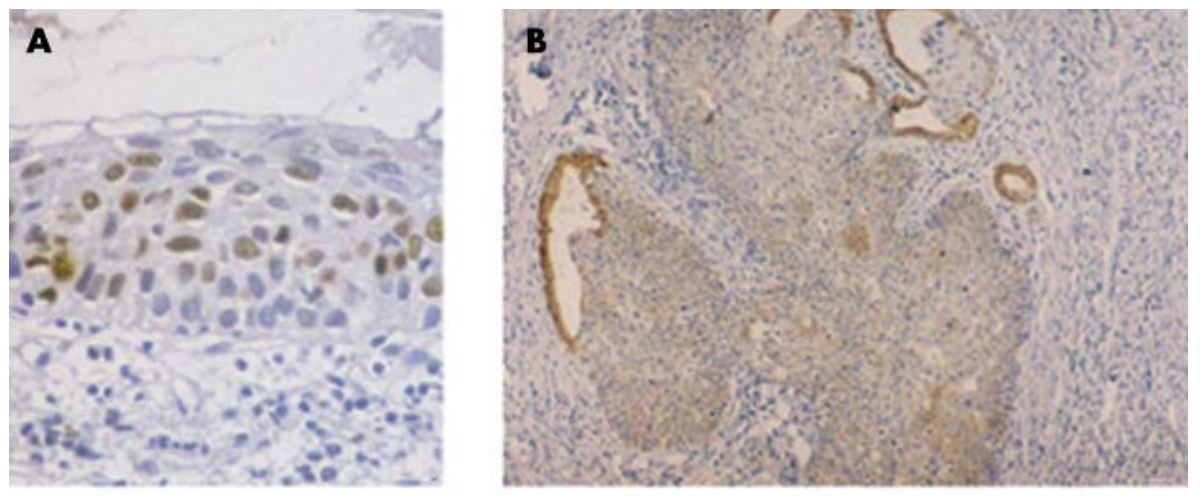

Figure 6 Immunohistochemical staining patterns in normal cervix and cervical lesions. (A) $\mathrm{Ki}-67$ staining in many nuclei of a cervical intraepithelial neoplasia (CIN) III lesion; (B) CIN III lesion expressing cytokeratin 8 (Ck8); endocervical glands also express $\mathrm{Ck} 8$; (C) normal cervix expressing $\mathrm{Ck} 13$ in the suprabasal layers; (D) CIN III lesion with loss of expression of $\mathrm{Ck} 13$; (E) normal cervix expressing Gp230 in the suprabasal layers; (F) CIN III lesion with loss of expression of Gp230.
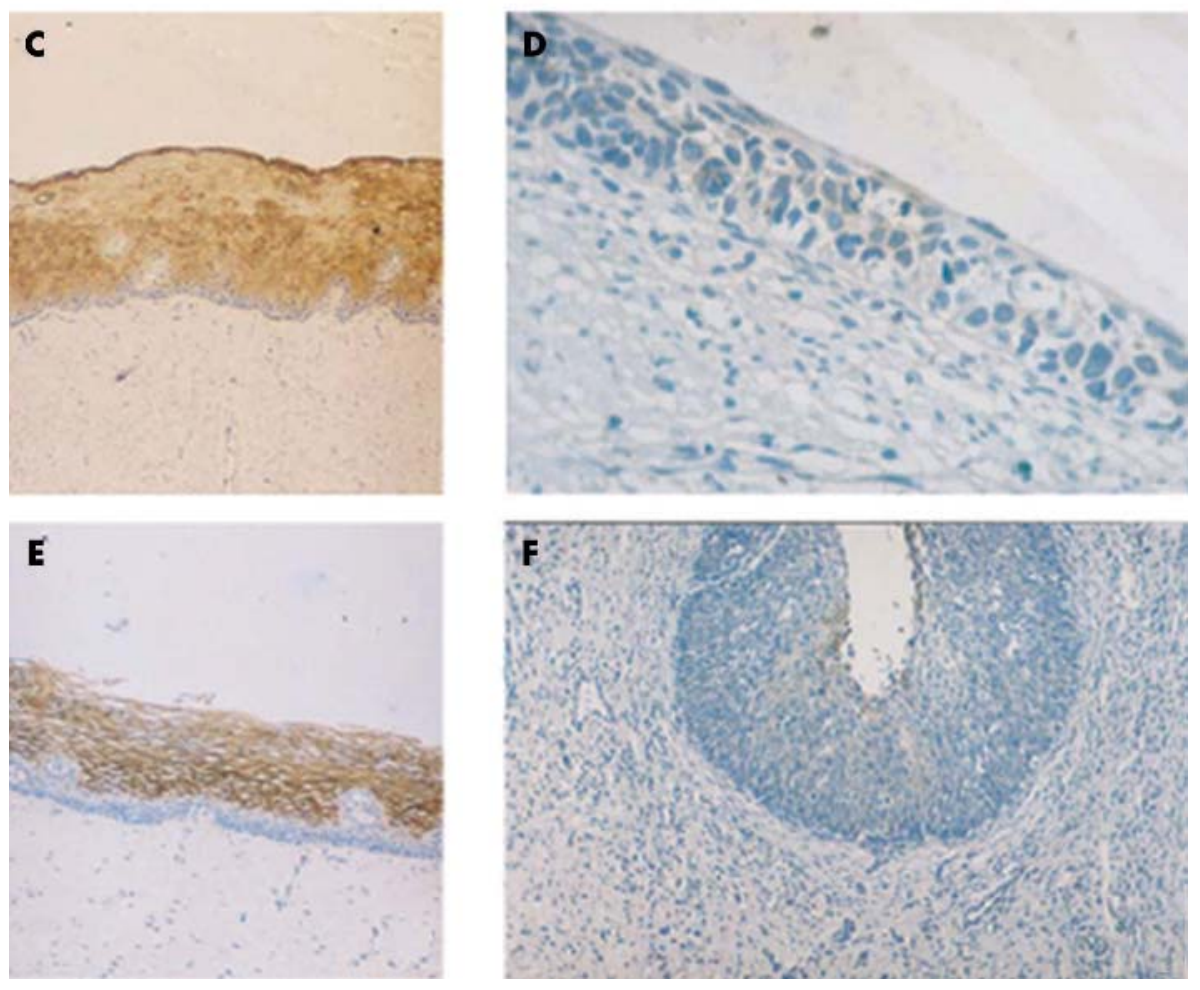

The small number of cases in our study does not allow us to make definitive conclusions regarding the frequency of multiple infections, and this should be confirmed by larger studies in the future. Despite this, the amount of information we collected should be taken into account for designing both primary prevention strategies, namely vaccines, and for building the best approach in screening programmes. ${ }^{24} 25$

\section{"We cannot rule out the possibility that at least a proportion of cervical intraepithelial neoplasia lesions progress in the absence of human papillomavirus"}

The low number of cases evaluated in our present series also does not allow us to make comparisons with previous studies with respect to the relative frequencies of viral types. In any case, the viral types we identified in our study (HPV types $16,33,35$, and 58 ) are the most frequently reported in African populations. ${ }^{2-6}$

As expected, we found a positive relation between the presence of HPV in the individual samples and the presence of CIN lesions. In fact, all the specimens in which HPV was detected showed CIN lesions. However, CIN lesions were also seen in HPV negative samples. We cannot exclude the possibility that the characteristics of the specimens (low representativity of neoplastic tissue, relatively low quality of the preservation method ( $10 \%$ unbuffered formalin), and possible low viral load) might explain the HPV negative results in our cases.

However, because most of the HPV negative samples had CIN lesions-mainly CIN III lesions-we cannot rule out the possibility that at least a proportion of CIN lesions progress in the absence of HPV, thus favouring the concept that CIN can be a bona fide neoplastic lesion. Supporting this interpretation are data showing that HPV is lost/disintegrated during cancer progression. ${ }^{26}$ Another explanation is the "hit and run concept". ${ }^{27}{ }^{28}$ We think that it is unlikely that our screening process missed a rare or even yet unknown viral type.

Although our study has obvious limitations regarding the number of cases (five), it is interesting that HPV-58 was always detected in samples with CIN III lesions (15 of 27) and HPV-35 was detected more frequently in samples with CIN I lesions ( 15 of 22). This observation may be partly explained by different capabilities of the E6 protein to inactivate $\mathrm{p} 53$ according to the type of high risk HPV. ${ }^{29}$

The high percentage of cells positive for Ki-67 and p53 in CIN III lesions agrees with previous reports. ${ }^{30-33}$ The similar degree of Ki-67 and p53 expression in HPV positive and 
negative samples seen in our study and in previous publications, ${ }^{2}{ }^{3033}$ is probably the result of the high prevalence of CIN lesions in HPV negative sections.

In previous studies, we evaluated the usefulness of different markers for the identification of preinvasive and invasive cervical lesions: Ck8, Ck10, Ck13, Ck17, Gp230 glycoprotein, and simple mucin-type carbohydrates-Tn, Sialyl-Tn, T, and Sialyl-T. ${ }^{20}{ }^{34}$ We identified the expression of $\mathrm{Ck} 8$ and $\mathrm{Ckl} 7$ and the loss of $\mathrm{CklO}$ and $\mathrm{Ckl} 3$ as good indicators of malignant transformation in the human cervix,$^{34}$ in agreement with other publications. ${ }^{19}{ }^{35-37}$ We also found that loss of expression of Gp230 glycoprotein is associated with malignant transformation at preinvasive stages. ${ }^{20}$ Investigation of the expression of simple mucintype carbohydrates showed that Tn and ST were useful markers of invasive carcinomas. ${ }^{20}$ In an attempt to expand our study in preinvasive cervical lesions and to map precisely their pattern of expression we evaluated their profile of expression in whole cervical cone biopsies.

The expression of $\mathrm{Ck} 8$ and $\mathrm{Ck} 17$ showed a complete (Ck8) or almost complete (Ck17) overlap with CIN III lesions, reinforcing their practical usefulness for the detection of CIN III lesions, namely in cytology specimens ${ }^{37}$ Loss of expression of both $\mathrm{CklO}$ and $\mathrm{Ck} 13$ is associated with preinvasive cervical lesions, more consistently for Ckl0. However, the lack of a consistent profile of alterations hampers their usefulness for diagnostic purposes.

Altered expression of Gp230 glycoprotein, Tn, and sialyl-T was frequently seen in all low grade and high grade cervical lesions, showing that mucin and mucin glycosylation changes are early events in cervical neoplasia, as reported previously by our group ${ }^{20}$ and by others. ${ }^{17}{ }^{38}$ However, the lack of consistency between their expression profile and the cervical lesions in our whole cone specimens limits their usefulness for diagnostic purposes. Altered expression of sialyl-Tn was not detected in our present series, in agreement with our previous report, ${ }^{20}$ showing that sialyl-Tn has little value as a marker for malignancy in the human cervix.

None of the putative diagnostic markers showed a clear association with HPV infection, indicating that they are markers of cervical lesions and not of cervical infection by HPV.

In conclusion, our study shows that when whole cone biopsies are evaluated the rate of infection by multiple HPV types is very high, and combines the most frequent high risk HPV types described in African populations. We also show

\section{Take home messages}

- We evaluated human papillomavirus (HPV) infection in five whole cervical cone specimens from Mozambican women with cervical intraepithelial neoplasia (CIN), together with the expression of cytokeratins, Ki-67, p53, Gp230, and simple mucin-type carbohydrates

- All patients showed high risk HPV types-namely types $16,33,35$, and 58 - and four of the five patients were infected by multiple viral types

- Thus, when whole cervical cone specimens are evaluated the rate of multiple HPV infection is very high

- The expression of cytokeratins 8 and 17 is a useful marker of CIN III

- The expression of Gp230 and simple mucin-type carbohydrates was altered in CIN but the lack of consistency limits their usefulness for diagnostic purposes that Ck8 and Ck17 are useful markers for CIN III lesions, independent of HPV infection.

\section{ACKNOWLEDGEMENTS}

This work was supported by Instituto de Cooperação Científica e Tecnológica Internacional (Cooperação com os PALOPs) and Fundação Calouste Gulbenkian.

\section{Authors' affiliations}

C Carrilho, M Alberto, L Buane, Department of Pathology, Medical School, Eduardo Mondlane University, and Maputo Central Hospital, Maputo, Mozambique

C Carrilho, L Cirnes, N Mendes, L David, IPATIMUP-Institute of Molecular Pathology and Immunology, University of Porto, Rua Roberto Frias, $s / n, 4200$, Porto, Portugal

L David, Medical Faculty of the University of Porto

\section{REFERENCES}

1 Bosch FX, Lorincz A, Munoz N, et al. The causal relation between human papillomavirus and cervical cancer. J Clin Pathol 2002;55:244-65.

2 Carrilho C, Gouveia P, Cantel M, et al. Characterization of human papillomavirus infection, P53 and Ki-67 expression in cervix cancer of Mozambican women. Pathol Res Pract 2003;199:303-11

3 Castellsague X, Menendez C, Loscertales MP, et al. Human papillomavirus genotypes in rural Mozambique. Lancet 2001;358:1429-30.

4 Mayaud P, Weiss HA, Lacey CJ, et al. Genital human papillomavirus genotypes in northwestern Tanzania. J Clin Microbiol 2003;41:4451-3.

5 Thomas JO, Herrero R, Omigbodun AA, et al. Prevalence of papillomavirus infection in women in Ibadan, Nigeria: a population-based study. Br J Cancer 2004;90:638-45.

6 Xi LF, Toure P, Critchlow CW, et al. Prevalence of specific types of human papillomavirus and cervical squamous intraepithelial lesions in consecutive, previously unscreened, West-African women over 35 years of age. Int $J$ Cancer 2003:103:803-9.

7 Bosch FX, Muñoz N, de Sanjosé S, et al. Human papillomavirus and cervical intraepithelial neoplasia grade III/carcinoma in situ: a case control study in Spain and Colombia. Cancer Epidemiol Biomarkers Prev 1993;2:415-22.

8 Schiffman MH, Baver HM, Hoover RN, et al. Epidemiologic evidence showing that human papillomavirus infection causes most cervical intraepithelial neoplasia. J Natl Cancer Inst 1993;85:958-64.

9 Cuschieri KS, Cubie HA, Whitley MW, et al. Multiple high risk HPV infections are common in cervical neoplasia and young women in a cervical screening population. J Clin Pathol 2004;57:60-72

10 Gravitt PE, Peyton CL, Alessi TQ, et al. Improved amplification of genital human papillomavirus. J Clin Microbiol 2000;38:357-61.

11 Quint WGV, Scholte G, van Doom U, et al. Comparative analysis of human papillomavirus infections in cervical scrapes and biopsy specimens by general SPF10 PCR and HPV genotyping. J Pathol 2001;194:51-8.

12 Bekkers RL, Melchers WJ, Bulten J, et al. Localized distribution of human papillomavirus genotypes in the uterine cervix. Eur J Gynaecol Oncol 2002;23:203-6.

13 Park JP, Sun D, Genest DR, et al. Coexistence of low and high grade squamous intraepithelial lesions of the cervix: morphologic progression or multiple papillomaviruses? Gynecol Oncol 1998;70:386-91.

14 Scully RE, Bonfiglio TA, Kurman RJ, et al. World Health Organisation: histological typing of the female genital tract tumors. Berlin: Springer Verlag, 1994.

15 Kjeldsen T, Clausen H, Hirohashi S, et al. Preparation and characterization of monoclonal antibodies directed to the tumor-associated O-linked sialosyl$2 \rightarrow 6 \propto-\mathrm{N}$-acetylgalactosaminyl (sialosyl-Tn) epitope. Cancer Res 1988;48:2214-20

16 Clausen H, Stroud M, Parker J, et al. Monoclonal antibodies directed to the blood group $A$ associated structure, galatosyl-A: specificity and relation to the Thomsen-Friedenreich antigen. Mol Immunol 1988;25:199-204.

17 Nielsen PA, Mandel U, Therkildsen MH, et al. Loss of a mucin-like epithelial glycoprotein in oral and cervical squamous cell carcinomas. Cancer Res 1997:57:634-40.

18 Hsu SM, Raine L, Fanger H. Use of avidin-biotin-peroxidase complex $(A B C)$ in immunoperoxidase techniques. A comparison between $A B C$ and unlabeled antibody (PAP) procedures. J Histochem Cytochem $1981 ; 29: 577-80$.

19 Smedts F, Ramaekers F, Link M, et al. Detection of keratin subtypes in routinely processed cervical tissue: implications for tumour classifications and the study of cervix cancer aetiology. Virchows Arch 1994;425:145-55.

20 Carrilho C, Cantel M, Gouveia P, et al. Simple mucin-type carbohydrate antigens (Tn, sialosyl-Tn, T and sialosyl-T) and gp 230 mucin-like glycoprotein are candidate markers for neoplastic transformation of the human cervix. Virchows Arch 2000;437:173-9.

21 Jacobs MV, van den Brule AJC, Snijders PJF, et al. A non-radioactive PCR enzyme-immunoassay enables a rapid identification of HPV 16 and 18 in cervical scrapes after GP5+/6+ PCR. J Med Virol 1996;49:223-9.

22 Jacobs MV, Snijders PJF, van den Brule AJC, et al. A general primer GP5+/ GP6+-mediated PCR-enzyme-immunoassay method for rapid detection of 14 
high-risk and 6 low-risk human papillomavirus genotypes in cervical scrapings. J Clin Microbiol 1997;35:791-5.

23 Levy JE, Fernandes S, Tateno AF, et al. Presence of multiple human papillomavirus types in cervical samples from HIV-infected women. Gynecol Oncol 2004;92:225-31.

24 Kuhn L, Denny L, Pollack A, et al. Human papillomavirus DNA testing for cervical cancer screening in low-resource settings. J Natl Cancer Inst 2000;92:818-25

25 Muñoz N, Bosch FX, Castellsagué X, et al. Against which human papillomavirus types shall we vaccinate and screen? The international perspective. Int J Cancer 2004;111:278-85.

26 Riou G, Favre M, Jeannel D, et al. Association between poor prognosis in early-stage invasive cervical carcinomas and non-detection of HPV-DNA. Lancet 1990;335:1171-4

27 Iwasaka T, Hayashi Y, Yokoyama M, et al. "Hit and run" oncogenesis by human papillomavirus type 18 DNA. Acta Obstet Gynecol Scand 1992;71:219-23.

28 Morgan D, Pecoraro G, Rosenberg I, et al. Human papillomavirus type $6 \mathrm{~b}$ DNA required for initiation but not maintenance of transformation of $\mathrm{Cl} 27$ mouse cells. J Virol 1990;64:969-76

29 Lechner MS, Laimins LA. Inhibition of p53 DNA binding by human papillomavirus E6 proteins. J Virol 1994;68:4262-73.

30 Bar JK, Harlozinska A, Sedlaczek P, et al. Relations between the expression of P53, c-erbB-2, ki-67 and HPV infection in cervical carcinomas and cervical dysplasias. Anticancer Res 2001;21:1001-6.
31 Hietala KA, Kosma VM, Syrjanen KJ, et al. Correlation of MIB-1 antigen expression with transcription factors Skn-1, Oct-1, AP-2, and HPV type in cervical intraepithelial neoplasia. J Pathol 1997; 183:305-10.

32 Kruse AJ, Baak JP, de Bruin PC, et al. Relationship between the presence of oncogenic HPV DNA assessed by polymerase chain reaction and $\mathrm{Ki}-67$ immunoquantitative features in cervical intraepithelial neoplasia. J Pathol 2001;195:557-62.

33 Mittal KR, Lin O, Chan W, et al. Cervical squamous dysplasias and carcinomas with immunodetectable p53 frequently contain HPV. Gynecol Oncol 1995;58:289-94.

34 Carrilho C, Alberto M, Buane L, et al. Keratins 8, 10, 13, and 17 are useful markers in the diagnosis of human cervix carcinomas. Hum Pathol 2004;35:546-51.

35 Maddox P, Sasieni P, Szarewski A, et al. Differential expression of keratins 10,17 , and 19 in normal cervical epithelium, cervical intraepithelia neoplasia, and cervical carcinoma. J Clin Pathol 1999:52:41-6.

36 Raju GC. Expression of the cytokeratin marker CAM 5.2 in cervical neoplasia. Histopathology 1988;12:437-43.

37 Martens J, Baars J, Smetds F, et al. Can keratin 8 and 17 immunohistochemistry be of diagnostic value in cervical cytology? A feasibility study. Cancer 1999;87:87-92.

38 Terasawa K, Furumoto $\mathrm{H}$, Kamada M, et al. Expression of Tn and sialyl-Tn antigens in the neoplastic transformation of the uterine cervical epithelial cells. Cancer Res 1996;56:2229-32. 